\title{
Smart Card-Based Infrastructure for Customer Data Collection and Handling in the IST E-Tailor Project (IST-1999-10549)
}

\author{
James Clarke $^{1}$, Stephen Butler ${ }^{1}$, George Kartsounis ${ }^{2}$, Simela Topouzidou ${ }^{2}$ \\ ${ }^{1}$ LAKE Communications, Business Innovation Centre, Ballinode, Sligo, Ireland \\ Jim.Clarke@lakecommunications.com \\ Stephen.Butler@lakecommunications.com \\ ${ }^{2}$ Athens Technology Centre, 6 Egialias Str., Paradisos, Maroussi, 151 25, Athens, Greece \\ Gkart@atc.gr, Simela@atc.gr
}

\begin{abstract}
The E-Tailor project (Integration of 3D Body Measurement, Advanced CAD, and E-Commerce Technologies in the European Fashion Industry (Virtual Retailing of Made-to-Measure Garments, European Sizing Information Infrastructure) is the largest project in the European Fashion Industry and one of the largest projects in the Information Society Technologies (IST) programme under the fifth framework programme of European research. E-TAILOR aims to develop advanced infrastructures, which will establish a new paradigm for Virtual retailing services of customised clothing (under Action Line (AL) II.3.3- personalisation of goods and services and AL IV.4.1 real-time clothing simulation and visualisation). In addition to presenting the ETailor project, this paper will concentrate on work being carried out in relation to innovative and secure Smart card applications being developed in ETAILOR
\end{abstract}

\section{Introduction}

A number of initiatives have arisen recently in many European countries, evolving around the concepts of Made to Measure Manufacturing and Retail shopping via the Internet. The combination of these new services is now possible by the emergence of technologies, systems and practices, such as 3D whole body scanners, automatic body measurement S/W, 3D CAD systems for the customisation of existing styles, Virtual try -on visualisation techniques and new smartcard technologies (multi-application Javacards)

The main objective of E-TAILOR is to develop a comprehensive innovative platform enabling the integration of new specialised forms of clothing retailing (Virtual-home shopping) with new high value-added services to the customer, namely the production of personalised garments at reasonable prices, in short time and with a close to perfect fit.

These new technologies promise a significant potential for the stimulation of the European Clothing Manufacturing and Retailing sectors; however, significant 
impediments must be removed in order to achieve a successful launching of the related applications and services.

E-TAILOR is founded on a global approach, global in terms of tackling all problems, global in terms of establishing a critical mass of all major players, global in terms of it $\mathrm{s}$ methodological approach combining both $\mathrm{R} \& \mathrm{D}$ and real state-of-the-art applications (demonstrators). Figure 1 contains a schematic of the entire E-Tailor system.

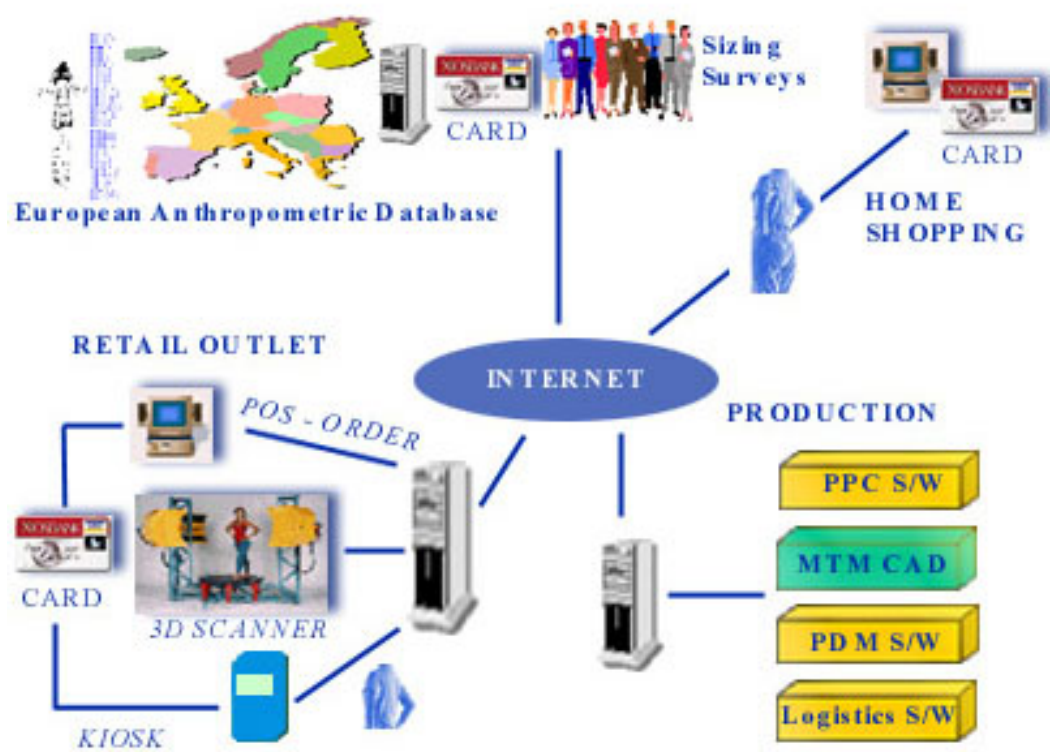

Fig. 1. E-Tailor Schematic Diagram

E-Tailor has the following technical/scientific objectives:

- To develop tools that will contribute to the solution of the sizing problem (nonuniformity of size designations, aggravated by the lack of updated body measurement data on European populations), in the form of a European Sizing Information Infrastructure (ESII). ESII will serve as a unification platform ensuring conformity in the storage and processing of sizing data, acquired in future full-scale sizing surveys, organised either by the member states or by the EU. The specifications for the content and data representation formats of a European Anthropometric Database (EAD) will be defined in common by the E-TAILOR User Group, prominent Clothing Institutes and the relevant standardisation committee (CEN/TC 248/WG 10, for the development of a standard size designation system of clothing).

ESII will consist of: 
- A European Anthropometric Database (EAD), linked to advanced tools for $3 \mathrm{D}$ body shape analysis, leading to the generalisation of body shapes and the production of a Library for Generic Body models of European populations.

- ESII will be embedded in a web-based Sizing Information Service for the European fashion industry.

- To develop a Customised Clothing Infrastructure (CCI), consisting of:

- intelligent design systems, based on rules and practices of human tailors (computer version of the tailor's brain)

- Order clustering and specialised ERP elements for the optimisation of the entire supply and production chain for overall quick response and cost effectiveness.

- CAD interoperability standards

- To develop a realistic and efficient clothing simulation and animation platform, accessible by kiosks and via Internet, and enabling the customers to visualise realistic models of themselves (obtained from 3D scanners), wearing "real" clothes with selected colours and accessories from the retailers collection (Virtual Try-on). This will tackle the need for specific interfaces required for Virtual shopping of clothing (the mirror or dressing room problem). The development of these tools will be based on existing state-of-the-art Virtual Reality systems used to dress and animate virtual mannequins. Further R\&D will enable:

- the dressing of customers 3D models with garment descriptions imported from CAD systems;

- the displaying of clothes with texture rendering different aspects of garments (such as fabric texture, seaming, buttons, zips, etc.);

- simple animation of the customers models, moving them to different positions, e.g. front, rear view, raising their arms, etc.

- To develop a generic 3D framework tackling the problem of inconsistent body measurements obtained from different scanners and related measurement software. Combined efforts of $3 \mathrm{D}$ scanner manufacturers and R\&D centres will lead to the development of:

- Generic body representations at various levels of symbolic abstraction for exportation to other applications, such as shape analysis, CAD systems and Virtual Try-on S/W;

- Generic representations of measurement data, as well as suggestions for standard EDI messages for the communication of measurement data;

- System independent measurement extraction S/W.

- To develop a comprehensive Customer Data Infrastructure (CDI) for the collection, storage and legitimate exploitation of customer data (including body measurements, personal preferences, etc.), thus tackling the problem of customer acceptance for the whole service and the problem of issuing multiple noninteroperable cards for the same customer.

- To integrate current state-of-the-art systems (3D scanners, MTM CAD, Virtual Shopping applications) in the form of across the value-added chain demonstrators. Demonstrators will, however, serve both as reference for the development of advanced systems, as well as testing and evaluation sites for new developments. 
The combination of R\&D and Demonstrators will thus result in a new paradigm for comprehensive Virtual retailing services of customised clothing.

Since the project is so large and covers a variety of areas, The E-Tailor project is split into three sub-projects, the European Sizing Information Infrastructure (ESII), The Customised Clothing Infrastructure (CCI) and the Virtual Shopping Infrastructure (VSI). The following three sections will explain the sub-projects in detail.

\section{Sub-project 1: The European Sizing Information Infrastructure (ESII) and Related Automatic Body Measurement Technologies (3D Scanners)}

\subsection{European Sizing Information Infrastructure (ESII)}

The actual situation of the different size systems utilised by European clothing industry and retailers is confusing and unsatisfactory. The size labeling is different from country to country. Up to now there is no common size system available in the European Community. As the current sizes are not comparable between the countries, industry and retailers face enormous problems when they want to export or import garments within the European Market. This is known as a huge impediment to the further development of the EC Market.

A system of standard sizes to be used in the European Community can only be established if there are qualified data available concerning the measures of the population. Some Member States carried out more or less reliable surveys. But mostly these surveys are very old and the reliability is not too good. Most of these data can not be used for the new common approach. New coordinated measurements have to be carried through.

A clothing technical committee has been set up in the CEN with the task to establish the future basis for a European standard size system. The target is to create a system which can be used European-wide with standardised size designations and after that to develop common size charts so that clothing fits to as many people as possible. This can only be defined if the CEN group has data available about the distribution of body proportions in the Member States.

In 1990, the European Commission stated in a paper that it would be useful to carry out a measurement survey of the European population in order to build up a system of size normalisation available for the clothing industry and valid for all Member States. $\square$ In the face of the upcoming new MTM production the necessity has increased enormously. The objectives of establishing an ESII are: -

- To develop a standardised framework and platform (infrastructure) for the deployment of a large scale European Sizing Survey (funded by external sources) for setting up a system of standard sizes to be used in the Member States 
- To set up a Web enabled European Anthropometric Database (EAD) in order to offer a new service to the European clothing industry and other interested industries

- To develop tools for standardised interpretation of anthropometric data (advanced shape analysis software, generic body models)

The ultimate target will be to organise and carry out a measurement survey in the European Community in order to get clear and extensive information about the distribution of the body measures in the population. A pilot technology proofing survey (approximately 1,000 persons) will test, populate and validate the European Anthropometric Database. The European Anthropometric Database (EAD) and the related innovative tools will offer a new service instrument to the European clothing industry, retailers, mail-order companies and other related industries.

\subsection{D Body Measurements and Systems}

Existing whole-body scanners permit fully automated sizing surveys and rapid individual measurements for custom clothing applications. However, the different 3D data acquisition techniques, the different scanned data formats and the varying measurement application software, result in inconsistent measurements across various systems (hardware and application software dependencies). The main objectives are: -

- To assess current systems (3D whole-body scanners, automatic measurement techniques) with respect to measurement consistencies;

- To develop standard representations for 3D raw data and measurement data, generic 3D body models, enabling the development of configurable interfaces to CAD systems, shape analysis and Virtual-Try-on $\mathrm{S} / \mathrm{W}$;

- To develop body measurement software, which is system independent, with the aim of achieving, as far as possible, unified and reproducible results obtained from different scanning systems.
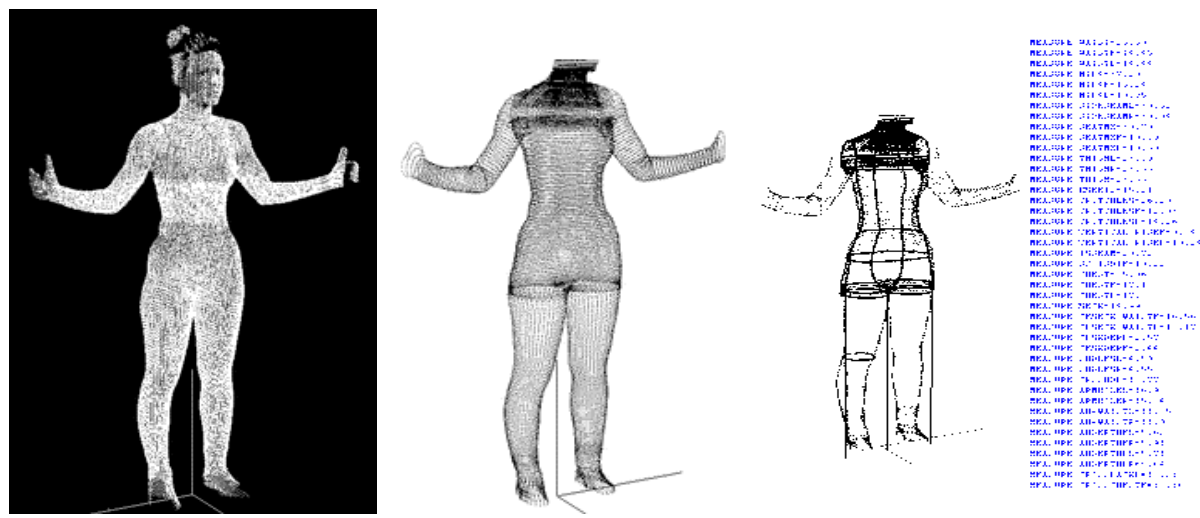

Fig. 2. From scanned data to measurements - Different body segmentation and body landmark detection techniques determine measurement results. 


\section{Sub-project 2: Customised Clothing Infrastructure}

The successful launching of Made-To-Measure services in the clothing industry is critically dependent on the following criteria:

- The fitting of the garments should be much better than the fitting of mass-produced clothing;

- The process for adapting existing styles to personalised measurements (alteration of existing patterns) is mainly based on geometrical transformations, based on the body measurements. However, in a true made to measure environment, only a handful of people have the skills and knowledge necessary to custom tailor a garment.

- The additional costs and delivery times involved in customisation. The whole ordering, design, production and logistics chain involves significant additional costs, compared to mass production. The client would not in general be prepared to pay more than an excess of $20 \%-30 \%$ to buy a custom-made garment. Furthermore, he would expect his order to be fulfilled in less than a week (3-4 days).

The processes that affect cost and delivery times are:

- Order handling processes. MTM orders are essentially unit orders;

- Communication processes;

- Design and production processes. Pattern alteration, marker making, cutting, sewing, finishing, packaging and delivery have to be done to the unit and, therefore have to be optimised.

- Raw material management is also critical. Fabric management must also be adapted to unit manufacturing.

The combination of these requirements lead to the need for the design and implementation of intelligent pattern alteration systems and an IT system (MTM ERP) to optimise the whole MTM supply chain so that:

- $\quad$ Fitting is close to what is expected from an experienced tailor;

- A fast response time (less than 4 working days) is achieved;

- A minimum Inventory of fabrics and other material is kept maintaining a good service level; and

- Machine set-up times and material handling are minimised.

\section{E-TAILOR's Tasks to meet the above requirements are:}

- The design and development of an intelligent pattern alteration system and a morphological editor, which will enhance the design and customisation capabilities of current MTM CAD systems;

- The development of critical components for a highly specialised (MTM focussed) Internet enabled ERP system. The following figure (fig.3) illustrates the main elements of an integrated MTM MRP.

- Suggestions for interoperability standards, which will enable the exchange of data between CAD systems of different suppliers. 


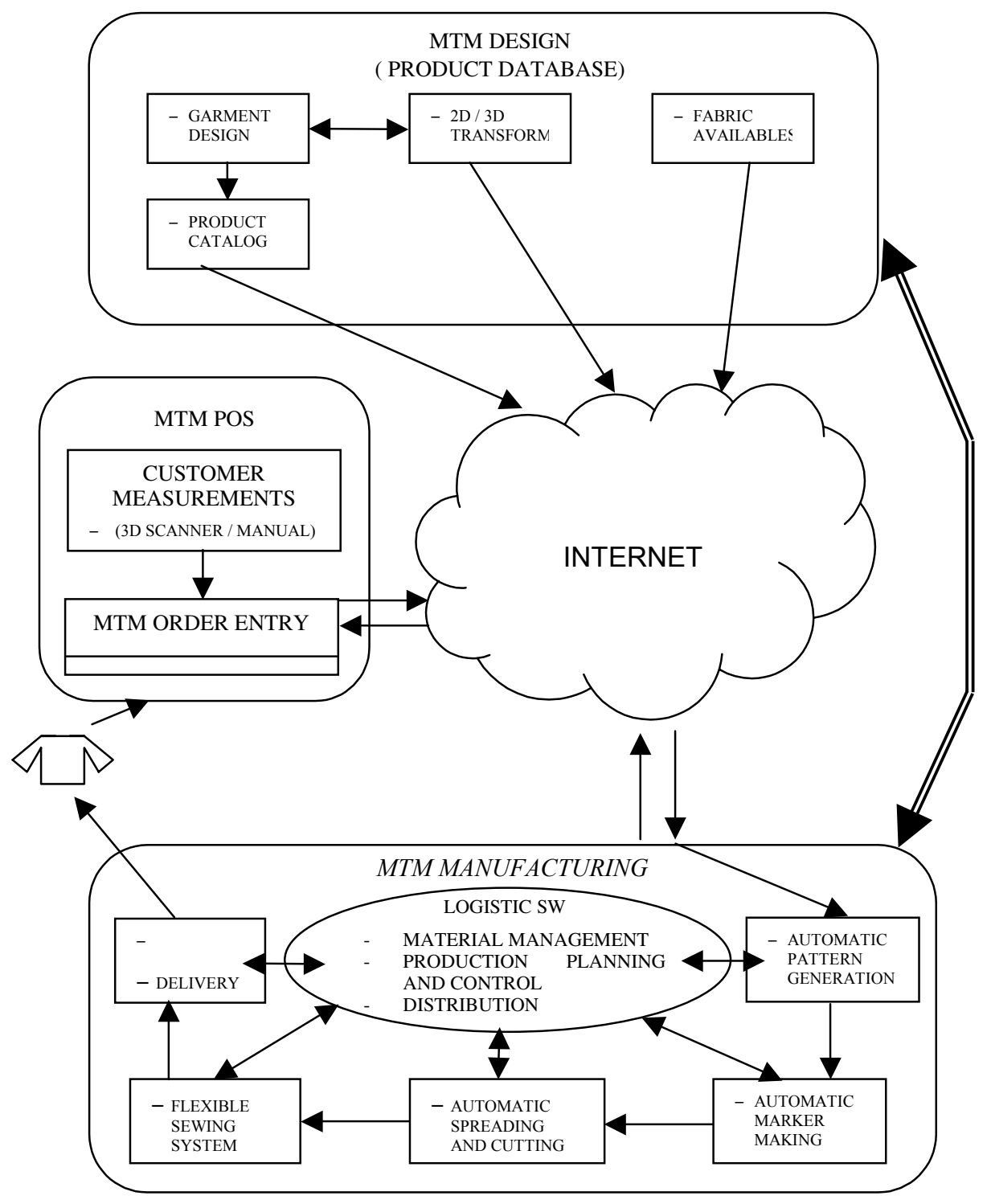

Fig. 3. E-Tailor MTM ERP Architecture

This approach requires the developments of interactive manipulation tools for $3 \mathrm{D}$ pieces of fabrics. The $2 \mathrm{D}$ garments are then imported into a $3 \mathrm{D}$ environment in two steps. 


\section{Sub-project 3: Virtual Shopping Infrastructure}

Most of the existing clothing simulation and animation systems are either nonrealistic, or too slow, they all require extensive user interaction and none of them permits clothing simulations on bodies of real humans, with real garments from the collection of a manufacturer, or retailer. Furthermore, data volumes and processing times do not permit simulations on the web.

\subsection{Objectives. The Objectives of SubProject 3 are:}

- To create a realistic and functional virtual shopping environment in the form of a comprehensive interactive service to the customers at retail kiosks and on the web. The core innovative tasks relate to the development of advanced clothing simulation techniques and software, enabling the customers to visualise realistic models of themselves (obtained from 3D scanners) wearing "real" clothes with selected colours and accessories from the retailers collection (Virtual Try-On software) and order with their customised smart cards;

- To integrate the Virtual Shopping Service with 3D scanners, CAD-CAM scannerWeb interfaces, Multi-application Smartcards and electronic catalogues.

The approach is to take the following as inputs: -

- 3D human body modelled with 3D scanners.

- 2D garments from clothes CAD/CAM software.

\section{To process the following: -}

- Assemble and seam the 2D garments to create a 3D garment;

- Dress the virtual mannequin with the 3D garment;

- Visualisation of the dressed mannequin for the web.

\section{Step 1. From geometric 2D patterns to 3D physics-based pieces of fabric}

This first step implies to import 2D CAD/CAM patterns, which only contain geometric and topological information (shape of the patterns, and seaming associations between patterns) and then convert them into 3D pieces of fabrics, which requires to convert the $2 \mathrm{D}$ patterns into a data structure that can be supported by the fabric mechanical model. The basic aspect of that process is constrained by the discretisation needed to perform the mechanical model simulation:
1 2D CAD/CAM importation
2 2D patterns discretisation
32 D patterns physical, texture, colour properties assignments

Step 2. Assembling fabrics and pieces on the virtual mannequin - automated mannequin dressing.

The approach is similar to the one used by tailors when they assemble 2D paper patterns onto a mannequin. They use pins to assemble the paper patterns directly onto the mannequin. For that purpose, there are some specific features (lines) on the mannequin that allow the tailor to know where to pin which pattern of paper. The 
result is a "dressed" mannequin, although the garment was directly assembled onto the mannequin. Similar features will be added onto the 3D scanned body in order to allow a direct placement of the $3 \mathrm{D}$ patterns on the mannequin. Once the garment is assembled and the mannequin is dressed, the physical simulation will give its precise shape to the garment, so that is fits to the mannequin it is attached to. Different postures of the mannequin can be chosen.

\subsection{Smart Card-Based Infrastructure for Customer Data Collection and Handling (CDI).}

\subsubsection{Introduction}

The smart card market in market in Europe has matured and grown significantly in the last few years. A number of proprietary electronic purses, telecommunications payment cards and loyalty schemes are available. There are a number of significant manufacturers of smart cards in Europe and hardware and software innovation continues rapidly. In Sub project 3 of the E-Tailor project, participants will develop and trial software/hardware components for the delivery of smart card mediated point of sale applications. It is foreseen with the widening of e-Commerce, the point of sale will move out of the retail unit and into the home, work place and public spaces through a variety of telecommunications enabled devices. It was decided to store on a smart card as the Secure Personal Data Storage (SPDS) because it will give a citizen full ownership and control of personal data, thus addressing privacy issues. An alternative would be to store the data in encrypted form and allow access by supplying a decryption protocol using a smart card.

The industry is now at the stage where it needs to converge on standards to gain widespread acceptance of the smart card by the citizen and the consumer. This section outlines some of the current options and standards available and makes some recommendations about an appropriate platform for E-Tailor personal data storage.

\subsubsection{Broad requirements of the Secure Personal Data Storage (SPDS).}

The requirements are broken into two categories - Customers and Retailers.

Customer $\mathrm{s}$ requirements of E-Tailor-enabled businesses: -

$>$ A technology that protects their ownership of personal ESII data;

- The ability to allow limited secure access to their data;

- Portability of their data from one point-of-sale context to another - retail kiosk to home PC connected to the web;

- Convenient inter-working with payment mechanisms and loyalty schemes;

- The data set will include 3-D body data, Front and side face images, Personal data, Private keys and Certificates;

- Customers are unlikely to accept anything greater than a 5 second delay for transfer of all of their data from the SPDS into the client application;

- It is likely that SPDS will be asked to return only certain parts of the data set so that some kind of indexed or tag-based file system will be necessary;

- Data stored on the SPDS should be protected from theft or misuse by someone other than the customer in two ways: 
a) The customer should own the SPDS and carry it on her person.

b) Data and applications on the SPDS should be protected by means of a pin or some form of biometrics protection (fingerprint, voiceprint, etc.).

Retailer $S$ requirements when providing E-Tailor body scanners: -

- A means of storing data extracted from a scanner;

- A way of associating this data with a customer;

- A means of assuring the customer of the privacy of her data;

- Standards-based software and hardware components to add to their scanner;

- In order to support structured and secured data access and hosting of applications, the SPDS should support multiple applications;

- It is of concern to all operators of an application distributed on smart cards that one application should not be able to corrupt of access data belonging to another application. The SPDS must support a security architecture that ensures the integrity and verifiable downloading of applications.

\subsubsection{Hardware/Software components of the Secure Personal Data Storage (SPDS).}

The CDI prototype hardware components will be cost-effective, secure and robust card readers that conform to chosen standards, which will utilise cost-effective multiapplication or secure data storage smartcards that conform to chosen standards.

The CDI prototype software components will include a generalized card-based application for the definition, access and modification of an XML-based ESII data structure on a multi-application smart card. We have selected XML because it is the obvious choice for the representation and manipulation (via a myriad of tools) of complex data structures with a high semantic content. The CDI components will provide enhanced access to this data structure to allow retailers limited access and customers full access since retailers and manufacturers will only need to know those aspects of body data essential for the manufacturing process. The rest of the data will be private to the citizen. The CDI will include standard software component proxies for a number of common smart card readers.

\subsubsection{Standards Compliance of the Secure Personal Data Storage (SPDS).}

The following standards are relevant to the work being carried out in the development of the E-Tailor SPDS: -

- Public key infrastructure - PKSC\#15

PKSC\#15 is a new proposed standard from RSA Inc. to deal with the problem of incompatible formats of smart cards for use in a Public Key Infrastructure.

- OCF - Open card Forum

OCF is intended to overcome the proprietary and complex nature of smart card reader programming.

- OpenCard architecture

To address the requirements and objectives described in $\mathrm{OCF}$, the core architecture of the OpenCard Framework features two main parts: theCardTerminal layer and the CardService layer. Further, the problem of card issuer independence is addressed separately by OCF's ApplicationManagementcomponent. 
- $\mathrm{PC} / \mathrm{SC}$ - PC Smart Card

The PC/SC Specifications 1.0 are completely platform independent, and can be implemented on any operating system.

- VOP - Visa Open Platform

Visa's open platform, which is now migrating to a multi-vendor standard as Global Platform, is an integrated environment for the development, issuance, and operation of multi-application smart cards. The standard consists of two parts - that covering the card terminal and that covering the smart card. The card specification defines an initial personalisation process and standard ways for customising the card after it has been issued. The goal is to provide tight control of the card and of the process of loading new applications onto the card.

- ISO 7816

The ISO 7816 standard "Identification cards - Integrated circuit cards with contacts" is the most significant standard for cards that establish electrical contacts with card readers when the card is inserted into the card slot.

- EMV ICC

EMV '96: ICC standards were agreed in 1996 and are commonly known as EMV. These are derived from ISO7816 and are intended by the sponsors (EuroPay, MasterCard \& Visa) to ensure interoperability in the payment business. They state a minimum functionality for smart cards and card terminals.

- Java Card

Java Card allows on-card applications to be written in Java, and at the same time provides a good base for multi-application smart cards. All the major smart card manufacturers have implemented a Java Card. The latest specification is version 2.1.

- Multos

The industry consortium Maosco, which owns the specification, owns Multos. Multos is a multi-application smart card OS which provides an ISO compliant file system interface and, in addition, and execution environment for custom applications. Application developers develop these applications in a new application language called MEL (Multos Executable Language).

- SmartCards for Windows

This card is a combination of the traditional ISO 7816-4 compliant operating system and a programmable platform. Windows for Smart Cards is an 8-bit, multiapplication operating system for smart cards with $8 \mathrm{~K}$ of ROM.

- CEPS and electronic purses

The 'killer application' that will drive the widespread introduction of multiapplication smart cards is the electronic purse. The electronic purse is a particular instance of a 'stored value' card, where monetary value, or something that can be equated to monetary value, such as loyalty points, electronic tickets etc., are stored on the card in a secure manner.

\section{Conclusions}

The research and development undertaken in E-Tailor will feed into Smart card-based products with the following competitive features: -

- Facilitate multi-application smart card applications for payment, transport, individual authentication etc. 
- Be portable across a wide range of hardware / software architectures including embedded devices and main-stream e-commerce applications

- Be portable across a wide range of point of sales venues - phones, PDA's, kiosks, public communications terminals and PCs

- Abide by emerging standards for smart cards, embedded systems and e-commerce applications to ensure maximum portability of our intellectual property.

\section{Acknowledgements}

The results described in this paper are an outcome of the work being carried out within the context of Information Society Technologies Programme (IST 199910549) E-TAILOR project, which is partially funded by the European Commission.

\section{References}

1. IST-1999-10549 E-Tailor. Deliverable Document 0201F01_D4Part1_5: T1.5 Personal Data Protection, July 2000. Authors - Hohenstein Institutes (DE), Somatometric Institute (EL), LAKE Communications (IRL), Nottingham Trent University (UK). 